\title{
Urge Urinary Incontinence and the Brain Factor
}

\author{
Carolyn Louise Morris* \\ Department of Geriatrics, University of Michigan, Ann Arbor, Michigan
}

\begin{abstract}
Aims: To evaluate cognitive processing in elderly women with urge incontinence as compared to continent cohorts by measuring performance on neuropsychological tests of working memory, attention and inhibition. To measure speed of information processing, intensity of bladder filling sensation, and urine volume at a first desire (FDV) and strong desire to void (SDV), and level of attentional demands. Methods: Test measures included Operation and Reading Span, Wisconsin Card-Sorting, Stroop Color Reading, Map Planning, and Maze Speed tests. A Likert scale measured bladder filling sensation intensity at FDV and SDV. An Attentional Demands Survey measured perceived attentional demands. Results: Women with urge incontinence had a mean severity index of $4.71 \pm 2.7$ with $84 \%$ demonstrating urge incontinence and $16 \%$ with mixed urge/stress incontinence. They experienced a FDV and a SDV to void at a lower bladder filling intensity with significantly smaller volumes of urine in the bladder compared to controls. Incontinent women also perceived significantly higher attentional demands but were not significantly different than controls on tests of executive function. Conclusions: While observations from neuroimaging provide evidence of differences between women with urge incontinence and controls in regional brain activity, efforts need to be made to decipher how these differences in metabolic activity relate to the fluid operations that create thoughts, decisions, and behavior. Neurourol. Urodynam. 32:441-448, 2013. @ 2012 Wiley Periodicals, Inc.
\end{abstract}

Key words: bladder control; brain; cognition; urgency; urinary incontinence

\section{INTRODUCTION}

The Problem

Urge incontinence, a multifactor disorder of bladder control during the storage phase, is characterized by frequency of urination, nocturia, an abnormal and compelling desire to void that is difficult to defer, and urinary incontinence. ${ }^{1}$ Adults with this disorder experience intermittent loss of voluntary control of their bladder and are usually incontinent of a moderate to large amount of urine.

\section{Control of Bladder Function}

In health, neural mechanisms that determine bladder control during the storage phase can be switched to the voiding phase either voluntarily or involuntarily (reflexively). ${ }^{2}$ Up until the age of 3 or 4, reflexive voiding is mediated by a spinobulbospinal pathway passing through the pontine micturition center (PMC) in the brainstem. ${ }^{2,3}$ When tension receptors in the bladder reach threshold, evidence suggests that afferent information is transmitted to the periaqueductal gray (PAG) in the midbrain where an excitatory signal is then relayed to the PMC. ${ }^{2,3}$ The PMC then sends a signal to pelvic nerves causing excitation of parasympathetic pathways and inhibition of somatic and sympathetic pathways that leads to relaxation of the urethral sphincter and pelvic diaphragm, contraction the bladder, and expulsion of urine. ${ }^{2,3}$

With increasing maturation of the nervous system, transition between phases (the storage or expulsion of urine) becomes voluntary, with evidence suggesting that the PAG relays afferent information from the bladder via the hypothalamus, thalamus, and insula to the anterior cingulate cortex (ACC) and prefrontal cortex (PFC) into the realm of conscious sensation. $^{3-5}$ The PFC, thought to have a role in attention and response selection, is believed to inhibit the PAG so that excitatory signals to the PMC are suppressed, thereby keeping the bladder in storage phase until the appropriate moment to void has been determined. ${ }^{3-5}$

\section{Clinical Studies and Brain Scans}

Development of a working model of brain activity has evolved from clinical studies (animal and human) and from a decade of functional brain imaging. Imaging studies provide evidence that the $\mathrm{PFC}$, and other subraspinal brain regions (PAG, insula, thalamus, hypothalamus, ACC) are metabolically active during bladder filling and emptying. ${ }^{5}$

During imaging, comparison of volunteers with urge incontinence to controls reveal that activity in the PFC is weaker in incontinent women and suggests some type of dysfunction in that region of the brain. ${ }^{6}$ Frequently neurological disease involves a failure to store urine, and lesions in the frontal lobe have been shown to produce bladder control characteristic of urge incontinence. ${ }^{4,7}$ Lesions in the PFC may alter the set point of the voiding reflex so that as the volume of urine in the bladder reaches a critical level, a switch to reflexive voiding occurs. ${ }^{3}$

Imaging studies reveal that metabolic activity in the insula is weaker with small bladder volumes and much stronger at high volumes in women with urge incontinence compared to controls. ${ }^{5}$ This difference in the activity of the insula may signify that incontinent women have poor sensory perception at low bladder volumes and are overly sensitive to bladder filling sensation at high volumes. ${ }^{5}$

Women with urge incontinence also demonstrate exaggerated brain activity in the ACC during imaging compared to controls. ${ }^{7}$ Empirical research on the role of the ACC in cognition provides evidence that the ACC is associated with tasks

Conflict of interest: none.

Eric Rovner led the peer-review process as the Associate Editor responsible for the paper.

${ }^{*}$ Correspondence to: Carolyn Louise Morris, MSN, PhD, University of Michigan, Department of Geriatrics, 4260 Plymouth Rd, Ann Arbor, Michigan

E-mail: clmorris@umich.edu

Received 29 May 2012; Accepted 30 October 2012

Published online 28 November 2012 in Wiley Online Library

(wileyonlinelibrary.com)

DOI 10.1002/nau.22357 
calling for the overriding of prepotent responses, tasks requiring the participant to choose among a set of equally permissible responses, and tasks that lead to a commission of errors. ${ }^{8}$ It is postulated that activation of the ACC and PFC co-occur with ACC activation occurring under circumstances where input from cognitive control is insufficient given current task demands. ${ }^{8}$

\section{Voluntary Behavior}

Voluntary control of bladder function depends on learned behavior that leads to the ability to postpone voiding until it is convenient and safe to do so with the right inferior frontal gyrus, part of the PFC, playing an important role in determining the appropriateness of social behavior such as micturition and the decision to void. ${ }^{3,4,7}$ Some patients with known frontal lesions show automatic "utilization" behaviors in which responses are dominated by the current context, suggesting that control has reverted to the external environment. ${ }^{9}$

Evidence suggests that the essential task of cognitive control (executive functions) is to overcome the prepotent "default mode" of automatic behavior. ${ }^{9}$ In order to override prepotent influences, a person must have the ability to inhibit attention to irrelevant stimuli, to maintain task set, and select choices in line with current goals. ${ }^{9}$ Cognitive control leading to voluntary behavior is theorized to depend upon neural networks in the PFC that involve a set of fluid operations/cognitive processes that enable intentional processing and adaptive cognitive performance. ${ }^{9,10}$

\section{Neural Networks}

Neural networks, patterns of interconnected neurons, are the "resource" that determine the quality of neural processing. ${ }^{10}$ These networks are theorized to create cognitive processes that serve as a "buffer" between stimulus and response and generate "thoughts" that loosen stimulus-response linkages. ${ }^{10}$ Cognitive processing is theorized to consist of working memory, attention, and inhibition; fluid operations that serve as the basis for the production of voluntary behavior. Working memory is theorized to create a delay period between the stimulus and the response. ${ }^{11}$ Attention is theorized to maintain the requisite level of neuronal activity during the delay period necessary to control the transformation of information so that it follows a neural path toward the desired response; while inhibition (the complement of attention) is theorized to suppress neural activity that might disrupt and interfere with the transformation of information and divert activity away from the desired goal. ${ }^{12}$ These cognitive operations are interrelated, and age-related changes that occur in one operation can have a substantial impact on the others.

\section{Aging Changes of the Brain}

Aging of the brain proceeds in a selective fashion with the PFC taking the heaviest toll, the parietal lobe showing greater resilience, and the pons being insensitive to the impact of aging. ${ }^{13}$ Cognitive processing has a sensitive dependence on cerebral blood flow, connectivity in neural networks, neurotransmitters, metabolic activity, and speed of processing, and a small change in any of them could result in radical differences in subsequent functioning of the PFC. ${ }^{13,14}$ Efficient cognitive functioning depends on the integrity of white matter, the density of synaptic connections, and on the specificity of synaptic pruning caused by interactions with the external environment. ${ }^{9}$ With the frontal lobes being the first to be impaired in aging, it is postulated that frontally mediated executive functions gradually decline and with this decline, there is difficulty overcoming prepotent automatic behavior influenced by the external environment with cognitive performance initiated in response to environmental contingencies. ${ }^{9,15-17}$

With aging, scientific evidence suggests that the inability to adequately activate the dorsolateral PFC disrupts behavioral performance. ${ }^{18}$ It is theorized that disruption of behavioral performance occurs because the activity level of task relevant neural networks fall below the threshold required to deal with the immediate perceptual and behavioral challenge or decreases to the point where activity of task irrelevant networks gain a competitive edge and interfere with information processing of moment. ${ }^{19}$ In the elderly, evidence indicates that there is recruitment of brain areas (selective and compensatory efforts) for specific tasks, which is believed to represent an effort to maintain functional brain integrity. ${ }^{20}$

\section{Life-Cycle Changes}

The aging process affects the structure and function of both the lower urinary tract and supraspinal neural networks necessary for control of bladder function and continence. The incidence of urge incontinence increases as people age. It is a time in the life-cycle when multiple physical, psychological, and social change occur, increasing demands on cognitive capacity, a limited cognitive resource. With increased demands on cognitive capacity, cognitive processing may decline and destabilize the ability to deal effectively with circumstances of the moment and the ability to function in daily life.

\section{Environmental Triggers: Influences of the Environment}

Many patients with symptoms of urge incontinence provide anecdotal information that entering their home (latchkey) or exposure to the sight, sound, or feel of running water (sensory stimuli strongly associated with urination) trigger a sensation of urgency and difficulty getting to the toilet without leaking urine. These anecdotes suggest that at times, prepotent automatic behaviors from thoughts below the level of conscious awareness are interfering with the "buffer" between stimulus and response.

\section{Study Perspective}

This study is carried out from within a framework of cognitive theory and is based upon both a working model of brain activity during bladder filling and emptying and symptoms and circumstances under which incontinence is experienced and described by patients with urge incontinence in clinical practice. The purpose of the study is to evaluate whether cognitive processes necessary for the creation of the "thought"/ decision to postpone voiding are altered or disrupted in some way, leading to the development of urge incontinence as people age. It is theorized that there may be some "intermittent" failure in cognitive processing that allows bladder control to falter and possibly revert to reflexive voiding. This is an exploratory study that evaluates one of the possible factors in this multifactor disorder and one of the areas in the brain, the PFC.

\section{Study Aims}

This study evaluates cognitive processing in elderly women with symptoms characteristic of urge incontinence as 
compared to continent cohorts by measuring performance on traditional neuropsychological tests of working memory, attention, and inhibition. A secondary aim was to compare measures of speed of information processing, intensity of bladder filling sensation, and urine volume at a first desire and a strong desire to void and level of attentional demands as perceived by study participants.

\section{METHODS}

\section{Study Volunteers}

Volunteers who responded to study advertisements were recruited with approval of the Institutional review Board (medicine) at the University of Michigan. After reminding volunteers that the study was recruiting women with both poor and good bladder control, women were screened for participation in the study by answering questions from a screening tool developed for the study (see addendum).

The convenience sample consisted of 100 women with symptoms of urge incontinence and 100 continent cohorts. Study participants had no overt neurological disease, were ages 60-79 years, right handed, and living in the community Exclusion criteria included a history of cognitive impairment; disease, injury, or surgical intervention of the central nervous system and/or spinal column; congestive heart failure, diabetes mellitus, hypercalcemia, untreated B12 or thyroid deficiency, alcoholism; bipolar depression, schizophrenia, or psychosis; radical pelvic surgery, chemotherapy, or radiation therapy to the pelvic area; use of medication known to affect cognition or bladder function; and a urinalysis that revealed a urinary tract infection, hematuria, or glycosuria. Inclusion criteria included a 12th grade education level or higher with the ability to read, write, and speak English, the ability to read newspaper print (both eyes, with correction), the ability to hear normal conversation, and demonstrate a Mini-Mental Status Examination (MMSE) score of 25 or greater. ${ }^{21}$ Incontinent women were included if they expressed symptoms of urgency, frequency, and nocturia accompanied by incontinence, demonstrated little or no urine leakage with $250 \mathrm{ml}$ of urine in the bladder while standing and coughing (standing stress test), demonstrated a post void residual less than or equal to $100 \mathrm{ml}$ after urinating, and expressed a willingness to discontinue medication for incontinence 2 weeks before and during participation in the study. Continent cohorts were included if they denied involuntary urine loss for 1 year prior to the study. Incontinent women and continent cohorts who met all study criteria were entered into the study after signing the study consent form.

\section{Measures/Instruments}

Incontinence Severity Index uses a four level index to measure incontinence severity and has been validated against a 48 hour pad weighing test. ${ }^{22}$ The index consists of two questions with severity scores ranging from 1 to 12 .

Span Tasks measure the attentional capacity necessary to control behavior by maintaining relevant information active in working memory while suppressing interference from information processing. ${ }^{23}$

Operation Span (OSPAN) (automated version) (http:// psychology.gatech.edu/renglelab/index.htm) is mouse driven, paced on the basis of each individual's time to complete mathematical operations, and is automatically scored upon completion. ${ }^{24}$ This span task requires participants to solve a series of simple mathematical operations while attempting to remember a list of unrelated letters in the order given. Reading Span (automated version) requires participants to read a set of sentences and determine whether they make sense or not while maintaining particular target letters in memory. Scores for both span tests range from 0 to 75 .

Wisconsin Card-Sorting Test (WCST) measures the ability of an individual to pay attention to an immediate goal and then shift the behavioral goal in response to changing circumstances while controlling impulsive responses and solving problems. ${ }^{25}$ Participants match each of the response cards to one of the four key cards and are given feedback when errors are made. The sorting principle changes after ten consecutive correct responses from color to form to number until all of the cards have been placed. ${ }^{26}$ The WCST has been used extensively in clinical and research applications as a measure of executive function (attention and inhibition), and evidence indicates that it is a sensitive measure of frontal lobe dysfunction. ${ }^{26}$ The version used in this study consisted of 128 trials. Computer Version 4 scoring program was used to automatically score the WCST, using normative data developed by Heaton, Chelune, Talley, Kay, and Curtiss (1993) for individuals ages $6.5-89$ years. ${ }^{27}$

Maze Tracing Speed Test and Map Planning Test measures the ability to look ahead and plan a course of action. ${ }^{28}$ During the Maze Tracing Speed Test, the participant must find their way through as many mazes as possible during 2 (3 min) trials. The test is scored as the total number of mazes traversed successfully. During Map Planning Test, the participant must find the shortest route between 2 points (while passing only one building) during 2 (3 min) trials. The test is scored as the total number of routes completed successfully.

Attentional Demands Survey measures attentional demands in four domains: physical environment (such as traversing indoor and outdoor obstacles), informational (such as dealing with medical jargon, font size in reading material), behavioral (such as transportation and mobility problems), and affective demands (such as feelings of loss and grief, worries and concerns). ${ }^{29}$ The survey consists of 42 items that require the respondent to determine the degree of effort or how difficult each domain makes life.

Stroop Color Dot Reading Task (Victoria version), a measure of speed of information processing, measures the ability to name the color of colored dots as quickly as possible. ${ }^{30}$ Scaled score equivalents (in seconds) by age groups 60-69 (65) and 70-79 (75) were used instead of raw scores. ${ }^{31}$

\section{Procedures}

The study required two visits in order to limit cognitive fatigue. During the 1st visit, measurements were taken of blood pressure, height, and weight for calculation of body mass index (BMI), and level of emotional affect/distress (Geriatric Depression Scale). ${ }^{32}$ Participants were asked to consume 16 ounces of water one-half hour prior to the first visit and drank water until they experienced and expressed a sensation of a first desire to void (FDV), "I feel like I might want to pass urine now," and a strong desire to void (SDV) "I am getting uncomfortable and would like to pass urine now,"33 (statements with permission, Abrams). FDV and SDV were measured using a Bladder Sensation Intensity Scale, which ranged from 0, no sensation of bladder filling, to 10, an intense sensation of a full bladder. A bladder scan was performed at a FDV to measure urine volume, and urine volume at a SDV was measured using a calibrated urine collection device. The following cognitive tasks were administered in randomized order: the Stroop 
Dot Color Reading Task, the Maze Tracing Speed Test, and the Map Planning Test. Incontinent women filled out an Incontinence Severity Index (with permission, Sandvick). All participants were instructed on how to fill out a 4-day voiding and fluid intake diary to be returned to the researcher on the second visit.

During the 2nd visit, the 4-day diary was reviewed with each participant. Operation Span and Word Span were administered in randomized order with administration of the Wisconsin Card-sorting test between them. Participants filled out a short Demographic Questionnaire between the 1st and 2nd cognitive tasks and filled out the Attentional Demands Survey (with permission, Jansen) between the 2nd and 3rd cognitive tasks. The questionnaire and survey were spaced in this way to allow a short break between the more arduous cognitive tasks.

\section{Analysis}

Statistical analysis was carried out using SPSS version 19. Independent sample $t$-tests were used to compare differences for group characteristics, measurement results from cognitive tasks, diary data, volume of urine at a FDV and a SDV, and attentional demands. Differences were considered to be statistically significant at $P<0.05$ (two-tailed). Pearson Chisquare was used to determine differences between groups for ethnicity and type of medical conditions. Pearson correlation was used to determine relationships between variables of interest.

\section{RESULTS}

Study participants had a mean age of 67 years (median age of 66.4) with a mean educational level of 15 years and were not significantly different for age, education level, ethnicity, Gravida/Para, blood pressure, number of medications, and medical conditions that would impact brain function (hypertension, cardiac disease, and depression; Tables I-III). Women with incontinence compared to continent women had a higher BMI $(\bar{X}=29.31 \pm 5.69$ vs. $\bar{X}=27.70 \pm 5.29 ; P=0.04$; effect size $=0.30$ ).

Sixteen percent of the women with incontinence demonstrated mixed urge/stress incontinence by leaking urine $(\bar{X}=1.12 \mathrm{ml} \pm 0.90$; range $0.20-3.75 \mathrm{ml})$ with approximately $250 \mathrm{ml}$ in the bladder while performing a standing stress test. $^{34}$ Incontinent women had a moderate level of incontinence severity with a mean score of $4.71 \pm 2.7$ with $61.2 \%$ of the sample with a score of 4 or less and $38.8 \%$ with a score of 6 or more, range 1-12 (Fig. 1).

Four-day averages from the diary revealed that women with incontinence compared to continent women had:

1. A lower volume of urine in the bladder at a FDV: $(\bar{X}=234.15 \mathrm{ml} \pm 116.55 \quad$ vs. $\bar{X}=334.36 \mathrm{ml} \pm 191.73$; $P=<0.0001$; effect size 0.63 ).
TABLE I. Demographic and Group Characteristics

\begin{tabular}{|c|c|c|}
\hline Bladder control & Mean & SD \\
\hline \multicolumn{3}{|c|}{ Age of subject (years) } \\
\hline Incontinent & 67.8 & 5.6 \\
\hline Continent & 67.4 & 6.0 \\
\hline \multicolumn{3}{|l|}{ Education level } \\
\hline Incontinent & 15.8 & 2.6 \\
\hline Continent & 15.9 & 2.5 \\
\hline \multicolumn{3}{|c|}{ Basal metabolic index } \\
\hline Incontinent & 29.3 & 5.7 \\
\hline Continent & 27.7 & 5.3 \\
\hline \multicolumn{3}{|c|}{ Systolic blood pressure } \\
\hline Incontinent & 138.7 & 17.8 \\
\hline Continent & 136.7 & 17.7 \\
\hline \multicolumn{3}{|c|}{ Diastolic blood pressure } \\
\hline Incontinent & 76.8 & 10.0 \\
\hline Continent & 75.1 & 11.5 \\
\hline \multicolumn{3}{|c|}{ Number of medications } \\
\hline Incontinent & 2.7 & 2.3 \\
\hline Continent & 2.3 & 2.0 \\
\hline \multicolumn{3}{|c|}{ Number of medical conditions } \\
\hline Incontinent & 2.0 & 1.4 \\
\hline Continent & 2.0 & 1.5 \\
\hline \multicolumn{3}{|c|}{ Number of pregnancies } \\
\hline Incontinent & 2.9 & 2.0 \\
\hline Continent & 2.5 & 1.7 \\
\hline \multicolumn{3}{|c|}{ Number of live births } \\
\hline Incontinent & 3.2 & 9.8 \\
\hline Continent & 2.0 & 1.4 \\
\hline
\end{tabular}

TABLE II. Bladder Control $\times$ Ethnicity

\begin{tabular}{|c|c|c|c|c|c|c|c|}
\hline & & \multicolumn{5}{|c|}{ Ethnicity } & \multirow[b]{2}{*}{ Total } \\
\hline & & Caucasion & Black & Hispanic & Asian & Other & \\
\hline \multirow{3}{*}{$\begin{array}{l}\text { Bladder } \\
\text { control } \\
\text { Total }\end{array}$} & Incontinent & 89 & 9 & 2 & 0 & 0 & 100 \\
\hline & Continent & 85 & 10 & 0 & 3 & 2 & 100 \\
\hline & & 174 & 19 & 2 & 3 & 2 & 200 \\
\hline
\end{tabular}

2. A lower volume of urine in the bladder at a SDV: $(\bar{X}=402.03 \pm 154.84$ vs. $478.89 \pm 203.395 ; P=0.004$; effect size $=0.37$ ).

3. A higher number of voiding episodes during waking hours $(\bar{X}=7.88+2.444$ vs. $\bar{X}=7.05+1.867 ; P=0.008$; effect size $=0.38$ )

4. A shorter voiding interval $(\bar{X}=2.54 \mathrm{hr} \pm 0.830$ vs. $\bar{X}=2.80 \mathrm{hr} \pm 0.877 ; P=0.032$; effect size $=0.30$ ) with the longest voiding interval being shorter as well $(\bar{X}=4.21 \mathrm{hr} \pm 1.071$ vs. $\bar{X}=4.69 \mathrm{hr} \pm 1.078 ; P=0.002$; effect size $=0.45$ ).

Type of medical condition

\begin{tabular}{|c|c|c|c|c|c|c|c|c|}
\hline & & & & & & & & \\
\hline & & None & Cardiac & Hypertension & Depression & Other & $\begin{array}{l}\text { Hypertension } \\
\text { and Depression }\end{array}$ & Total \\
\hline Bladder control & Incontinent & 11 & 3 & 29 & 11 & 36 & 10 & 100 \\
\hline & Continent & 16 & 1 & 27 & 12 & 32 & 12 & 100 \\
\hline Total & & 27 & 4 & 56 & 23 & 68 & 22 & 200 \\
\hline
\end{tabular}




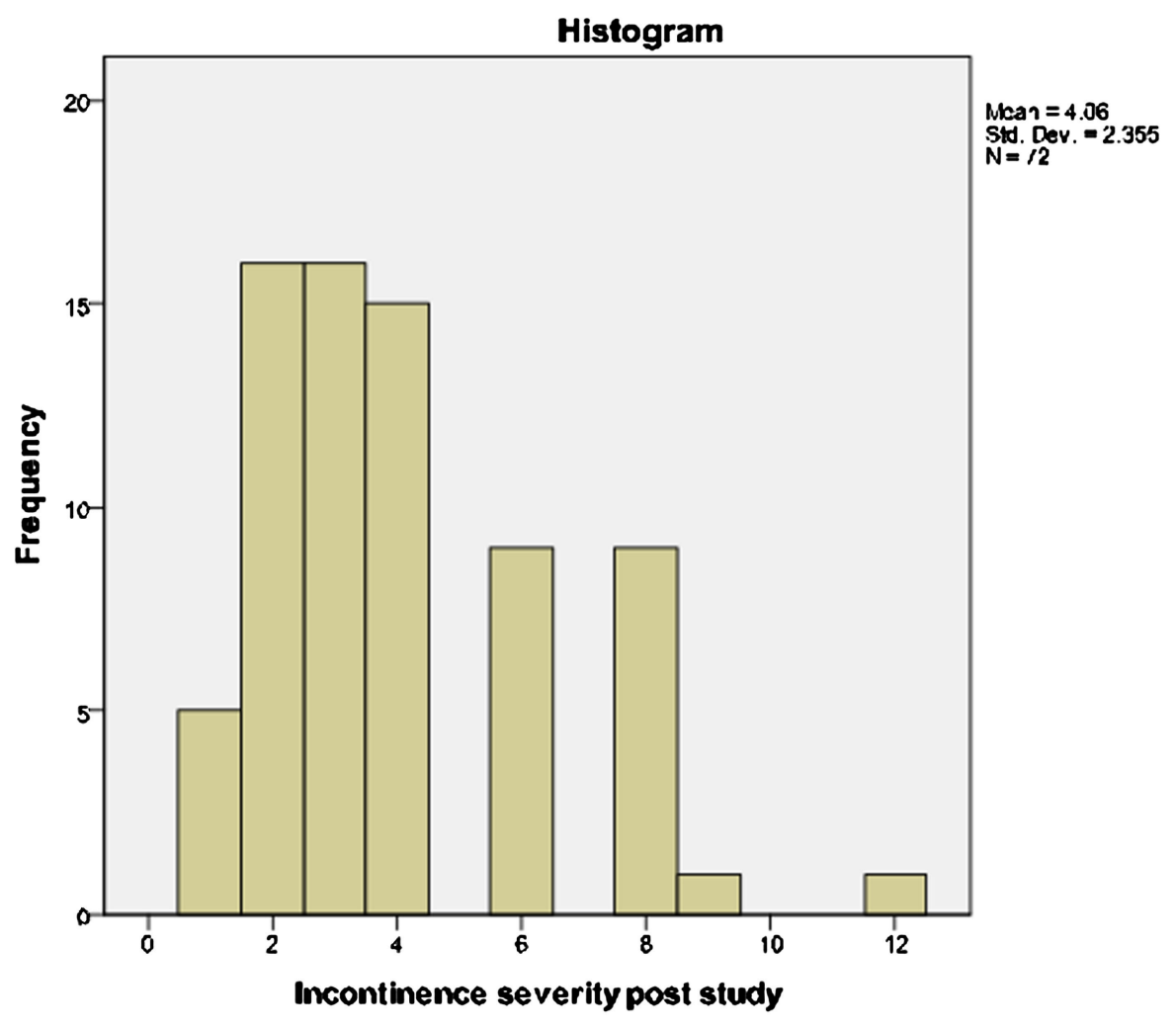

Fig. 1. Urge urinary incontinence and the brain factor.

There were no significant differences between groups for total fluid intake, total voided urine volume, or bladder capacity, or for the quantity of caffeinated, carbonated, or alcoholic beverages consumed.

Fifty-eight percent of incontinent women leaked during the 4-day diary $(\bar{X}=1.03 \pm 1.32$; range $0-8$ times). On the Bladder Sensation Intensity Scale, incontinent women experienced bladder filling intensity with a mode of 5 at a FDV and a mode of 8 at a SDV compared to continent cohorts with mode of 8 at a FDV and a mode of 9 at a SDV.

The Attentional Demands Survey revealed that women with incontinence compared to continent women experienced significantly more demands in the Affective Domain (emotion, preoccupations, and worries) that serve as sources of distraction $(\bar{X}=15.33 \pm 8.20$ vs. $\bar{X}=12.34 \pm 8.08 ; \quad P=0.01$; effect size $=0.37$ ) and a significantly higher Total Attentional Demands score $(\bar{X}=60.17 \pm 27.46$ vs. $\bar{X}=51.94 \pm 25.5$; $P=0.03$; effect size $=0.32$ ) as a whole.

The Stroop Color Dot Test, a test for speed of processing, the Maze Tracing Speed and Map Planning tests, tests of planning and looking ahead, Reading and Operation Span tasks, tests of working memory, and the Wisconsin Card-Sorting test, a test of attention to the immediate goal and ability to shift goal focus did not reveal a significant difference between groups. Since $64 \%$ of the sample was between the ages of 60-69 years, analyses were carried out to determine if the broad range of ages (60-80) was a factor for the negative results from cognitive testing. Groups of women older than 65 were compared and results remained negative for significant differences. The sample was then divided into age quartiles: ages 60-64 ( $n=35$ INC and 44 Cont); ages 65-69 ( $n=28$ INC and 20 Cont); ages $70-74$ ( $\mathrm{n}=21$ INC and 19 Cont); and ages $75-80$ ( $\mathrm{n}=16$ INC and 17 Cont) with the same negative results (Table IV for detailed age comparison details).
Correlation analysis for incontinent women revealed a weak relationship between domains from the Attentional Demands Survey with variables from the 4-day diary and volume of urine at a FDV and a SDV (Table V). For continent women, correlation analysis revealed that domains from the Attentional Demands Survey did not correlate with variables from the 4-day diary or with the volume of urine at a FDV, but bladder volume at a SDV did correlate weakly with attentional demands in the physical domain (Pearson correlation $=.207$; $0.046^{*}$ : sig at the 0.05 level.

\section{DISCUSSION}

As expected incontinent women compared to cohorts voided more frequently, at shorter intervals, with lower bladder filling intensity, and at lower urine volumes. Unexpectedly, the average consumption of caffeinated, carbonated, and alcoholic

\section{TABLE IV. Age Characteristics}

\begin{tabular}{lcc}
\hline Age & Incontinent & Continent \\
\hline & & \\
N Valid & & \\
$\quad$ Missing & 100 & 100 \\
Mean & 0 & 0 \\
Median & 67.8 & 67.4 \\
Mode & 67.7 & 65.1 \\
STD & $61^{*}$ & $60^{*}$ \\
Variance & 5.6 & 6.0 \\
Range & 31.9 & 36.3 \\
Minimum & 20 & 19 \\
Maximum & 60 & 60 \\
\hline
\end{tabular}

${ }^{*}$ Multiple modes exist. The smallest mode is shown. 


\begin{tabular}{|c|c|c|c|c|c|}
\hline \multirow[b]{2}{*}{ Bladder diary } & \multicolumn{3}{|c|}{ Four-day diary/attentional demands } & \multicolumn{2}{|c|}{ Incontinent group bivariate } \\
\hline & Total score & $\begin{array}{l}\text { Affective } \\
\text { domain }\end{array}$ & $\begin{array}{l}\text { Physical environment } \\
\text { domain }\end{array}$ & $\begin{array}{l}\text { Informational } \\
\text { domain }\end{array}$ & $\begin{array}{c}\text { Behavioral } \\
\text { domain }\end{array}$ \\
\hline \multicolumn{6}{|c|}{ Average daily number of voids } \\
\hline $\mathrm{N}$ & 98 & 98 & 98 & 98 & 98 \\
\hline Pearson correlation & $0.223^{*}$ & 0.106 & 0.189 & $0.263^{* *}$ & $0.216^{*}$ \\
\hline Sig. (two-tailed) & 0.027 & 0.298 & 0.063 & 0.009 & 0.033 \\
\hline \multicolumn{6}{|c|}{ Average daily voiding interval } \\
\hline $\mathrm{N}$ & 97 & 97 & 98 & 98 & 97 \\
\hline Pearson correlation & -0.185 & -0.112 & -0.128 & -0.181 & $-0.244^{*}$ \\
\hline Sig. (two-tailed) & 0.068 & 0.271 & 0.211 & 0.075 & 0.021 \\
\hline \multicolumn{6}{|c|}{ Volume of urine at a first desire to void } \\
\hline $\mathrm{N}$ & 98 & 98 & 98 & 98 & 98 \\
\hline Pearson correlation & $-0.210^{*}$ & $-0.213^{*}$ & $-0.231^{*}$ & -0.106 & -0.143 \\
\hline Sig. (two-tailed) & 0.038 & 0.035 & 0.022 & 0.297 & 0.159 \\
\hline \multicolumn{6}{|c|}{ Volume of urine at a strong desire to void } \\
\hline $\mathrm{N}$ & 98 & 98 & 98 & 98 & 98 \\
\hline Pearson correlation & $-0.218^{*}$ & $-0.307^{* *}$ & -0.193 & -0.093 & -0.134 \\
\hline Sig. (two-tailed) & 0.033 & 0.002 & 0.059 & 0.366 & 0.195 \\
\hline \multicolumn{6}{|c|}{ Longest average daily voiding interval } \\
\hline $\mathrm{N}$ & 98 & 98 & 98 & 98 & 98 \\
\hline Pearson Correlation & $-0.213^{*}$ & -0.139 & -0.171 & $-0.205^{*}$ & $-0.223^{*}$ \\
\hline Sig. (two-tailed) & 0.035 & 0.171 & 0.092 & 0.043 & 0.027 \\
\hline Sig. (two-tailed) & 0.035 & 0.171 & 0.092 & 0.043 & 0.027 \\
\hline
\end{tabular}

*Correlation is significant at the 0.05 level (two-tailed).

** Correlation is significant at the 0.01 level (two-tailed).

fluids were not significantly different between groups. It has been surmised that these types of fluid can have irritative and diuretic effects that contributes to bladder control problems.

Attentional demands were perceived as being significantly greater by women with incontinence than cohorts. While attentional demands correlated with diary data, volume of urine at a FDV and a SDV, $<10 \%$ of the variability in one variable was explained by the variability in the other variable. Cimprich $^{35}$ developed the Attentional Function Index (AFI) which she describes as a tool designed to measure perceived effectiveness in performing common activities requiring attention and working memory. In the future, the AFI might be helpful in assessing cognitive function of patients with urge incontinence.

In an earlier pilot study ${ }^{36}$ with a sample size of 20 participants per group, women with urge incontinence compared to continent cohorts were significantly slower on speed of information processing (Stroop Color Dot test) and were twice as likely and significantly different in their ability to pay attention to the card sorting category of the moment (failure to maintain set) during the WCST. Surprisingly, this study did not reveal significant differences between groups for any of the traditional tests of cognitive function. In an attempt to make sense of the negative results, information provided by patients in clinical practice will be reviewed, and the discussion will reflect current concepts of cognitive processing and bladder control while speculating on possible reasons for these results.

Patients frequently give a history that incontinence occurs "intermittently," "independent" of the volume of urine in the bladder, or the length of "time" since the last voiding episode. Frequently, they reveal that urgency occurs when they are exposed to environmental triggers, stimuli strongly associated with urination such as latch/key and water. Some women give a history of being in control of bladder function when in an "unsafe" social situation, but experience episodes of incontinence at home.
Fowler and Griffiths ${ }^{5}$ state that "it is believed that, for voiding to occur, the PMC requires an excitatory signal from the PAG and a "safe" signal from the hypothalamus." p. 52.

The bladder switches from the storage to the voiding phase when it is not "safe" to do so in people with urge incontinence, suggesting that possibly (at times) bladder control reverts to reflexive voiding. It is not safe to void while doing dishes, when walking into one's home, or before reaching the toilet or lowering one's clothes to toilet. It is very difficult to intentionally and voluntarily void in an "unsafe" environment as was demonstrated by participants, trained to void in a scanner, half of whom were unable to do so on command. ${ }^{5}$

It would appear that a decision was not made to delay voiding nor was there a decision to void in these "unsafe" situations. The ability to delay voiding until it is safe to do is postulated to require a "decision" so that the PFC sends a signal to the PAG to suppress the PMC. Interference from an intermittent increase in cognitive demands, ill-timed thoughts, and subconscious associations with toileting, and/or anxiety or fear of incontinence could disrupt cognitive processing and the ability to select a goal to delay voiding.

Maybe the "goal" is switched from a decision to delay voiding to the "thought" of rushing to the toilet before it is too late because repetitive experiences with incontinence cause patients to learn that they cannot prevent the bladder from emptying. Competing behavioral goals such as whether to try to delay voiding or whether to rush to the toilet may weaken control of bladder function and activity in the PFC because of "cross talk" during parallel cognitive processing, leading to increased activity in the ACC because of the conflict in goal selection and anxiety/fear of incontinence when input from cognitive control is insufficient given current task demands.

Emotions are an integral part of a person's internal state and, thus, have profound influences on the choices one makes. ${ }^{37}$ Chronic use of sensory neural modulation (SNM) has been successful in treating incontinence and suggests that 
neuroplastic changes that occur during chronic treatment may provide patients with a learned reaction to successful control of bladder function, renewing their belief in their ability to delay voiding until it is safe to do so. ${ }^{5}$

Based on the findings in this study, it is possible that traditional tests of executive function are not useful instruments to measure clinically significant cognitive deficits. They are tests that were initially developed to measure hypothesized constructs in experimental psychological investigations, and people with documented frontal lobe lesions have been found to perform well on these measures of cognitive function. ${ }^{38} \mathrm{Al}-$ exander and Stuss ${ }^{39}$ and Burgess et al. ${ }^{38}$ build a strong case for the development and use of novel clinical measures that correspond to conditions encountered in the "real" world and that predict problems experienced there.

Advisors to the study suggested the broad age range (60-79 years) because of difficulty recruiting women 70 and older in reasonably good health. This was a self-selected sample, and the most frequent reason for ineligibility for the study was a diagnosis of diabetes mellitus. While women in their 60's experience urge incontinence, the phenotype may be different than for women 70 and older. It is possible that a large sample of women in the 7th and 8th decade of life might demonstrate significant differences in cognitive function between groups.

Another possibility is that significant differences between women with urge incontinence and controls might be found if the cognitive load was increased by carrying out neuropsychological testing under challenging circumstances: while participants are being scanned and infused with a slow intravenous drip until bladder filling levels reach a sensory level defined as a first desire and SDV. Urgency may actually be a SDV intensified by fear of incontinence that causes the patient to feel an "urgent" desire to rush to the bathroom.

In a small sample $(n=20)$, a pilot study ${ }^{31}$ did not reveal a significant difference between groups during the Victoria version of the Stoop test possibly because successful mastery of ink-color naming (a less habituated response) instead of word reading (a dominant response) was reinforced during the incongruent block after a few trials with repeated practice. A large block of congruent trials with an occasional incongruent stimulus might be a better test of the ability to suppress/ inhibit a prepotent response and test the ability to focus attention to the goal at hand.

\section{CONCLUSION}

The essential task of executive function, neural processing involving working memory, attention, and inhibition, is to allow the person to overcome prepotent automatic behavior and attend selectively to the task goal of the moment. While this study did not identify evidence of impaired cognitive control, incorporating cognitive theory, and neuropsychological testing of cognitive processing into current research efforts might further the development of the current proposed model of bladder control. While observations from neuroimaging provide evidence of differences between women with urge incontinence and controls in regional brain activity, efforts need to be made to decipher how these differences in metabolic activity relate to the fluid operations that create thoughts, decisions, and behavior.

\section{ACKNOWLEDGMENTS}

In addition to acknowledging Dr. Edward McGuire, would you please add the Geriatric Center Clinics at the University of Michigan for their support of this project.

\section{ADDENDUM: TELEPHONE SCREENING O}

1. Spelling of name

2. Age

3. Home phone number

4. Are you right handed:

5. Do you have and 12th grade or higher education:

6. Do you have a history of stroke, Parkinson's disease_, head injury__, seizure disorder__, dizzy spells from circulatory problems of the brain__, spinal stenosis_or spinal injury_, memory problems, congestive heart failure___, diabetes mellitus__, high blood calcium_, an untreated $\mathrm{B} 12 \ldots$ or thyroid condition

7. Do you $\overline{\text { drink }}$ alcohol: __ ? Type of alcoholic bever-

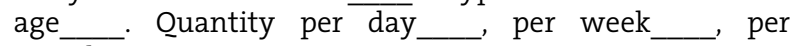
month

8. Do you have a history of any type of emotional disorder: ? Description

9. Do you have any history of brain___ or spinal cord surgery___, radical pelvic surgery___ chemotherapy radiation therapy__ or surgery for bladder_ rectal__ or vaginal problems___, or had surgery for urine leakage ?

10. What medication do you take?

11. Do you take over-the-counter sleeping aids or decongestants

12. Have you had any involuntary urine leakage/wetness during the last 12 months:

13. How often do you have unwanted urine leakage

14. Do you leak urine when you cough, sneeze, or exert yourself

15. Do you experience a strong need/urge to empty your bladder but cannot get to the bathroom or get your cloths down before getting wet from urine ?

16. Do you suddenly feel a need to rush to the toilet when you hear water running, put your hands in water, or when you walk into the house after being away

17. Do you empty your bladder more than 6 times during the day

18. Do you empty your bladder more than 2 times at night

19. Are you receiving any kind of treatment for urine leakage: ? Describe

20. Do you take medication for bladder control problems: ?

21. Would you be able/willing to stop the bladder control medication for 2 weeks in order to participate in this study:

22. Does your vision allow you to read the newspaper $?$

23. Do you have any trouble seeing colors:

24. Do you have any trouble hearing ? Description

25. Do you have any difficulty reading understanding English:

\section{REFERENCES}

1. Abrams P, Cardozo L, Fall M, et al. The standardization of terminology of lower urinary tract function: Report from the standardization sub-committee of the International Continence Society. Am J Obstet Gynecol 2002;187:116-26.

2. deGroat WC. Central nervous system control of micturition. In: Pat D, O'Donnell MD, editors. Urinary incontinence. St Louis: Mosby; 1997. p. 33-48.

3. Fowler CJ, Griffiths D, deGroat WC. The neural control of micturition. Nat Rev Neurosci 2008;9:453-66.

4. Fowler CJ. Integrated control of lower urinary tract-clinical perspective. Br J Pharmacol 2006;147:S14-24.

5. Fowler CJ, Griffiths DJ. A decade of functional brain imaging applied to bladder control. Neurourol Urodyn 2010;29:49-55. 
6. Griffiths D, Tadic SD. Bladder control, urgency, and urge incontinence: Evidence from functional brain imaging. Neurourol Urodyn 2008;27:466-74.

7. Andrew J, Nathan P. Lesions of the anterior frontal lobes and disturbances of micturition and defaecation. Brain 1964;87:233-62.

8. Botvinick $M$, Carter $C$, Braver T, et al. Conflict monitoring and cognitive control. Psychol Rev 2001;108:624-52.

9. Craik FIM, Bialystok E. Cognition through the lifespan: Mechanisms of change. Trends Cogn Sci 2006;10:131-8.

10. Fuster JM. Network memory. Trends Neurosci 1997;20:451-9.

11. Baddeley A. Working Memory. Science 1992;255:556-9.

12. Redick TS, Heitz RP, Engle RW. Working memory capacity and inhibition Cognitive and social consequences. In: Gorfein DS, MacLeod CM, editors. Inhibition in cognition. Washington D.C.: American Psychological Association; 2007. pp. 125-42.

13. Raz N. Aging of the brain and its impact on cognitive performance: Integration of structural and functional findings. In: Craik FIM, Salthouse TA, editors. The handbook of aging and cognition, 2nd edition. Mahwah, New Jersey: Lawrence Erlbaum Associates; 2000. pp. 1-90.

14. Raz N, Lindenberger U, Rodrique KM, et al. Regional brain changes in aging healthy adults: General trends, individual differences and modifiers. Cereb Cortex 2005;15:676-89.

15. L'Hermitte F. Human anatomy and the frontal lobes: Part II. Patient behavior in complex and social situations: The 'environmental dependency syndrome'. Ann Neurol 1986;19:335-43.

16. West RL. An application of prefrontal cortex function theory to cognitive aging. Psychol Bull 1996;120:272-92

17. Mesulam $M$. The human frontal lobes: Transcending the default mode through contingent encoding. In: Stuss D, Knight R, editors. Principles of frontal lobe function. New York: Oxford University Press; 2002. p. 8-30.

18. Jonides J, Nee DE. Brain mechanisms of proactive interference in working memory. Neuroscience 2006;139:181-93.

19. Goldman-Rakic PS. Architecture of the prefrontal cortex and central executive. In: Grafman J, Holyoak KJ, Boller F, editors. Structure and functions of the human prefrontal cortex, 2nd edition. New York; The New York Academy of Sciences: 1995. pp. 71-83.

20. Reuter-Lorenz PA, Stanczak L, Miller AC. Neural recruitment and cognitive aging: Two hemispheres are better than one, especially as you age. Psychol Sci 1999;10:494-9.

21. Tombaugh TN, Hubley AM, McDowell I, et al. Mini-Mental State Examination (MMSE) and the Modified MMSE (3MS): A psychometric comparison and normative data. Psychol Assess 1996;8:48-59.

22. Sandvik H, Seim A, Vanvik A, et al. A severity index for epidemiological surveys of female urinary incontinence: Comparison with 48-hour padweighing tests. Neurourol Urodyn 2000;19:137-45.
23. Unsworth N, Engle RW. Individual differences in working memory capacity and learning: Evidence from the serial reaction time task. Mem Cognit 2005;33:213-20.

24. Unsworth N, Heitz RP, Schrock JC, et al. An automated version of the operation span task. Behav Res Methods 2005;37:498-505.

25. Spreen O, Strauss E. A compendium of neuropsychological tests. New York: Oxford University Press; 1991.

26. Heaton RK, Chelune CJ, Talley JL, et al. Wisconsin card sorting test (WCST) manual revised and expanded. Lutz, Fl: Psychological Assessment Resources; 1993

27. Goldin JN. Wisconsin Card Sorting Test: Computer version 4 scoring program research edition. 4th edition. Lutz, Fl: Psychological Assessment Resources, Inc.; 2005.

28. Ekstrom RB, French JW, Harman $\mathrm{HH}$, et al. Mauual for kit of factorreferenced cognitive tests. Educ Test Serv 1976; Available from: http://www ets.org//Media/Research/pdf/Kit_of_Factor-Referenced_Cognitive_Tests.pdf.

29. Jansen D, Keller M. An instrument to measure the attentional demands of community-dwelling elders. J Nurs Meas 1999;7:197-214.

30. Stroop JR. Studies of interference in serial verbal reactions. J Exp Psychol $1935 ; 18: 643-61$

31. Troyer AK, Leach L, Strauss E. Aging and response inhibition: Normative Data for the Victoria Stroop Test. Aging Neuropsychol Cogn 2006;13: 20-35.

32. Sheik JI, Yesavage JA. Geriatric Depression Scale (GDS): Recent evidence and development of a shorter form. In: Brink TL, editor. Clinical gerontology: A guide to assessment and intervention. New York: The Haworth Press; 1986. pp. 165-74.

33. Abrams P. Urgency the key to defining the overactive bladder. BJUI 2005; 96:1-3.

34. Miller JM, Ashton-Miller J, DeLancey JOL. Quantification of the cough-related urine loss using the Paper Towel Test. Obstet Gynecol 1998;91:705-9.

35. Cimprich B, Visovatti $M$, Ronis DL. The Attentional Function Index-a self report cognitive measure. Psychooncology 2011;20:194-202.

36. Morris CL. Differences in the function of the prefrontal cortex between women with urge incontinence and continent cohorts (unpublished doctoral dissertation). Ann Arbor: University of Michigan; 2007.

37. Paulus MP, Yu AJ. Emotion and decision-making: Affect-driven belief systems in anxiety and depression. Trends Cogn Sci 2012;16:476-83.

38. Burgess PW, Alderman N, Forbes $C$, et al. The case for the development and use of "ecologically valid" measures of executive function in experimental and clinical neuropsychology. J Int Neuropsychol Soc 2006;12:194-209.

39. Alexander $M$, Stuss DT. Frontal injury: Impairments of fundamental processes lead to functional consequences. J Int Neuropsychol Soc 2006;12 $192-3$. 